\title{
A Grammar of the
}

Great Andamanese Language An Ethnolinguistic Study

Anvita Abbi

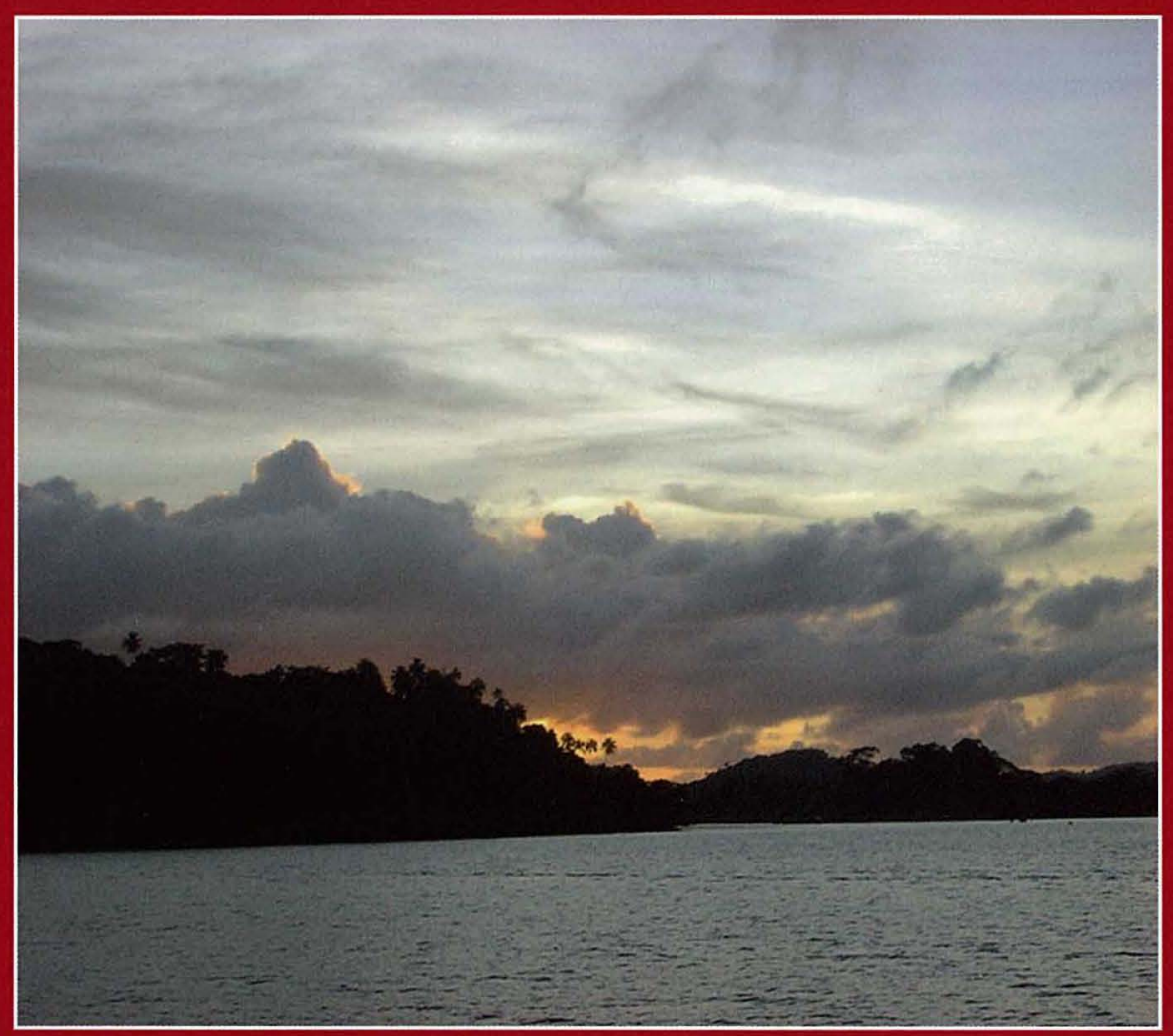

B R I L L 
A Grammar of the Great Andamanese Language 


\title{
Brill's Studies in South and Southwest Asian Languages
}

\author{
Series Editors \\ John Peterson, University of Kiel \\ Anju Saxena, Uppsala University
}

Eitorial Board

Anvita Abbi, Jawaharlal Nehru University

Balthasar Bickel, University of Zurich

George Cardona, University of Pennsylvania

Carol Genetti, University of California, Santa Barbara

Geoffrey Haig, University of Bamberg

Gilbert Lazard, cNRs \& École Pratique des Hautes Études Harold F. Schiffman, University of Pennsylvania

Udaya Narayana Singh, Visva-Bharati, Shantiniketan, India

VOLUME 4

The titles published in this series are listed at brill.com/bssal 


\title{
A Grammar of the Great Andamanese Language
}

\author{
An Ethnolinguistic Study
}

By

Anvita Abbi

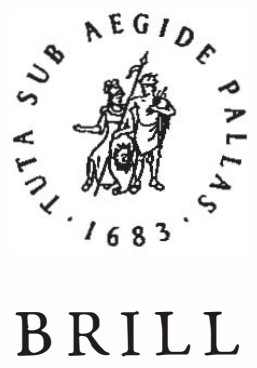

LEIDEN • BOSTON

2013 
Cover illustration: Strait Island, Andaman Islands. Picture courtesy of the author.

Library of Congress Cataloging-in-Publication Data

Abbi, Anvita, 1949-

A grammar of the great Andamanese language : an ethnolinguistic study / By Anvita Abbi. pages cm. - (Brill's studies in South and Southwest Asian languages ; 4)

Includes bibliographical references and index.

ISBN 978-90-04-23527-4 (hardback : alk paper) — ISBN 978-90-04-24612-6 (e-book)

1. Andamanese language-Grammar. 2. Andamanese language-Etymology. 3. EthnologyIndia-Andaman Islands (India) 4. Andaman Islands (India) - Languages. I. Title.

PL7501.A6A34 2013

$495 \cdot 9-\mathrm{dc} 23$

2013018345

This publication has been typeset in the multilingual "Brill" typeface. With over 5,100 characters covering Latin, IPA, Greek, and Cyrillic, this typeface is especially suitable for use in the humanities. For more information, please see www.brill.com/brill-typeface.

ISSN $1877-4083$

ISBN 978-90-04-23527-4 (hardback)

ISBN 978-90-04-24612-6 (e-book)

Copyright 2013 by Koninklijke Brill NV, Leiden, The Netherlands.

Koninklijke Brill NV incorporates the imprints Brill, Global Oriental, Hotei Publishing, IDC Publishers and Martinus Nijhoff Publishers.

All rights reserved. No part of this publication may be reproduced, translated, stored in a retrieval system, or transmitted in any form or by any means, electronic, mechanical, photocopying, recording or otherwise, without prior written permission from the publisher.

Authorization to photocopy items for intemal or personal use is granted by Koninklijke Brill NV provided that the appropriate fees are paid directly to The Copyright Clearance Center, 222 Rosewood Drive, Suite 910, Danvers, MA 01923, USA.

Fees are subject to change.

This book is printed on acid-free paper.

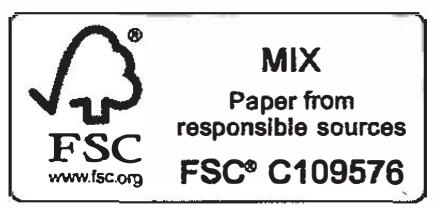

Printed by Printforce, the Netherlands 
To Peter Austin 



\section{CONTENTS}

Acknowledgements ......................................................................................... Xv

Preface .................................................................................................................................... xvii

List of Maps, Figures and Tables .................................................................. x xxi

Abbreviations and Symbols .................................................................................. $\quad$ xxv

I. The Andaman and the Great Andamanese

Introduction .............................................................................................................. 1

1.1 Geography and Topography _........................................................... 1

1.2 Population ................................................................................................. 3

1.3 Present-Day Great Andaman and the Great Andamanese $\quad . . .6$

1.3.1 Great Andaman .................................................................... 6

1.3.2 The Strait Island ...................................................................... 7

1.4 History of Language Studies .............................................................. 9

1.4.1 Great Andamanese ............................................................... 9

1.4.2 Study in Pre-Independent India $\quad$...................................... 12

1.4.3 Study in Post-Independent India .................................... 14

1.5 Genealogical Classification .......................................................... 14

1.6 Typological Differences .................................................................... 16

1.7 The Last Decade .................................................................................. 16

1.8 A Sociolinguistic Sketch of the Great Andamanese .............. 18

1.8.1 Background of the Great Andamanese Speakers $\quad . . . . . \quad 20$

1.8.2 Language Competence .......................................................... $\quad 22$

1.8.3 Language Use ........................................................................... 24

1.8.4 A Complex Situation ................................................................. 27

1.8.5 The Predicament .................................................................. 28

1.9 The Great Andamanese Culture: Some Observations .......... 29

1.9.1 Naming a Person ................................................................... 29

1.9.2 Naming Seasons: The Honey Calendar .......................... 30

1.9.3 Measuring Time in Great Andamanese ........................ 31

1.9.3.1 Parameters of Temporal Categorisation ...... 31

1.9.3.2 Natural Time Parameters ................................. 31

1.9.3.3 Evolutionary Period (Mythological Time) $\quad \ldots \quad 32$

1.9.3.4 Historical Parameters

(Pre-and Post-British Era) ............................ $\quad 32$

1.9.3.5 Life Cycle (Age of a Person) .............................. $\quad 32$ 
1.10 The Beliefs of the Great Andamanese ...................................... 33

1.10.1 The Domain or Realm .................................................. 34

1.10.2 Major Life Forms and Ethno-Biological

Classifications ....................................................................... 34

1.11 The Biological Universe of the Great Andamanese ............ $\quad 35$

$1.12 \quad$ The Present Study ............................................................................... $\quad 36$

II. Phonetics and Phonology

Introduction ........................................................................................................... 37

2.1 Vowels .......................................................................................... 37

2.1.1 Phonemic Contrasts $\quad$............................................................... $\quad 38$

2.1.1.1 Front Vowels ......................................................... 38

2.1.1.2 Back Vowels ............................................................ 39

2.1.2 Length ........................................................................................ 39

2.1.3 Phonotactics of Vowels $\quad$.................................................... 40

2.1.4 Vowel Sequences/Clusters ............................................... 41

2.2 Semi-Vowels/Glides ........................................................................ 44

2.3 Consonants ......................................................................................... 46

2.3.1 Phonemic Contrasts: Minimal and Sub-Minimal

Pairs for Consonants ........................................................ 49

2.3.1.1 Nasals ....................................................................... 49

2.3.1.2 Oral Stops _........................................................... 49

2.3.1.3 Fricatives ............................................................... 52

2.3.1.4 Liquids ..................................................................... $5^{2}$

2.3.2 Phonotactics of Consonants ......................................... 53

2.3.3 Medial Consonant Clusters ……...................................... 54

2.3.4 Initial Consonant Clusters $\quad$................................................ $\quad 56$

2.4 Syllables ............................................................................................... 56

2.4.1 Possible Syllable Structures $\quad$.............................................. 57

2.4.2 Constraints ............................................................................ 58

2.4.3 Length of a Word ................................................................... 58

2.5 Morphophonemics ....................................................................... 59

2.5.1 Gemination ............................................................................ 59

2.5.2 Degemination and Compensatory Lengthening $\quad \ldots \quad 59$

2.5.3 Homorganicity ...................................................................... 60

2.5.4 Insertion of a Consonant ................................................ 60

2.5.5 Vowel Harmony ................................................................. 60

2.5.6 Vowel Lowering ................................................................... 60

2.5.7 Vowel Deletion .......................................................................... 61

2.5.8 Syllable Attraction ............................................................. 61

2.5.9 Metathesis ................................................................................. 62

2.6 Acoustic Study of Problematic Sounds $\quad$...................................... 62 
III. Grammar Overview

3.1 The Structure

3.1.1 General

65

3.1.2 Typological Background .................................................. $\quad 65$

3.1.3 Core Arguments ................................................................... 67

3.2 Ambivalence of Verbs, Adjectives and Nouns ..................... 70

3.2.1 Noun Verb Ambivalence ................................................. 70

3.2.2 Adjectives as Verbs .......................................................... 74

3.2.3 Adjectives as Adverbs .................................................... 74

3.2.4 Other Unusual Features ................................................... $\quad 75$

3.3 Inalienability (INA), Body Division Classes and

Grammaticalisation ........................................................................ 76

3.3.1 Introduction .............................................................................. $\quad 76$

3.3.2 Anthropocentrism ............................................................... 77

3.3.3 The Semantics of Inalienability .................................. $\quad 78$

3.3.4 Linguistic Manifestations of 'Inalienability' ............. $\quad 79$

3.3.5 Body Class Markers and Other Nouns ……................ 81

3.4 The Semantic Role of Body Division Classes ....................... 81

3.5 Inalienability and its Representation in Verbs ..................... $\quad 82$

3.6 Inalienability and its Representation in Modifiers ............ 84

3.7 Process of Grammaticalisation ................................................. 85

3.8 The Status of Inalienability Markers in the Grammar:

Proclitics

3.8.1 Arguments for Treating Inalienability Markers as

Clitics

3.8.2 Nature of Proclitics .......................................................... 90

3.8.3 Clitic Sequencing ............................................................... 92

3.9 Summary and Conclusions …………............................................ 94

IV. Word Formation Processes

Introduction ........................................................................................... 97

4.1 Affixation .................................................................................... 97

4.2 Attaching Clitics ............................................................................... 100

4.2.1 Proclitics .................................................................................... 100

4.2.2 Enclitics ................................................................................ 102

4.2.3 Object Clitics _......................................................................... 102

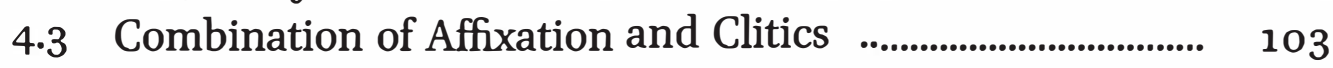

4.4 Compounding .................................................................................. 104

4.4.1 Formation of Compounds .............................................. 104

4.4.1.1 Noun + Modifier ................................................. 105

4.4.1.2 Noun + Noun ...................................................... 105 


\begin{tabular}{|c|c|}
\hline 4.4.1.3 & Noun + Verb or Verb + Noun \\
\hline 4.4.1.4 & Noun + Proclitic + Noun \\
\hline 4.4.1.5 & Noun + Proclitic + Verb/Modifier \\
\hline 4.4.1.6 & $\begin{array}{l}\text { Combination of Proclitics, Affixes, } \\
\text { and Compounds }\end{array}$ \\
\hline 4.4.1.7 & Antonyms \\
\hline 4.4.1.8 & Summary of Compounds \\
\hline
\end{tabular}

V. Nouns and Noun Phrases

Nouns

5.1 Number and Gender ................................................................. 112

5.1.1 Numerals ............................................................................................. 114

5.1.2 Gender ......................................................................... 115

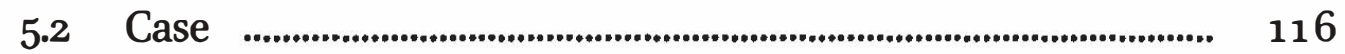

5.2.1 Arguments ....................................................................... 116

5.2.2 Case Markings ........................................................................... 117

5.2.2.1 Intransitive Subject Marking $\quad$........................ 117

5.2.2.2 Transitive Subject Marking and Ergative/ Agentive ................................................................. 119

5.2.2.3 Object Marking ….................................................. 120

5.2.2.4 Dative ...................................................................... 122

5.2.2.5 Instrumental .................................................... 122

5.2.2.6 Ablative ........................................................ 124

5.2.2.7 Comitative .................................................................... 125

5.2.2.8 Purposive/Benefactive ................................. 126

5.2.2.9 Directional ....................................................... 126

5.2.2.10 Locational .................................................................. 127

5.2.2.11 Genitive ......................................................... 130

5.2.2.12 Comparative .................................................... 131

5.3 Noun Phrases ................................................................................. 131

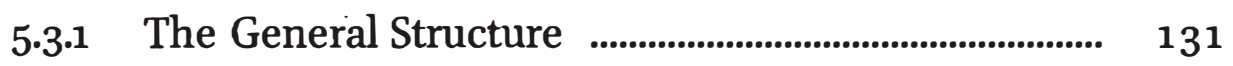

5.3.1.1 Descriptive .................................................... 132

5.3.1.2 Possessive ............................................................. 132

5.3.1.3 Appositional .................................................... 133

5.3.1.4 Quantifiers ..................................................... 133

5.3.1.5 Relativisation ...................................................... 133

5.3.2 Coordinated Noun Phrases .............................................. 134

5.4 Conclusion .................................................................................... 135 
VI. Possession

Introduction

137

6.1 Possessive Classification ............................................................... 137

6.2 Primary Possession .......................................................................... 138

6.2.1 Body Part Terms ......................................................................... 139

6.2.1.1 Mouth Cavity $(a=) \quad$............................................. 140

6.2.1.2 Major External Body Parts $(\varepsilon r=\sim e r=) \quad \ldots \quad 141$

6.2.1.3 Extremities of the Body $(u \eta=\sim o \eta=) \quad \ldots . . . \quad 142$

6.2.1.4 External Body Products or Extension

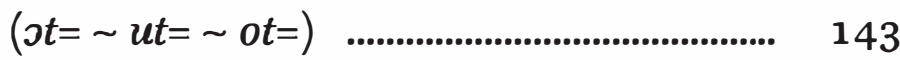

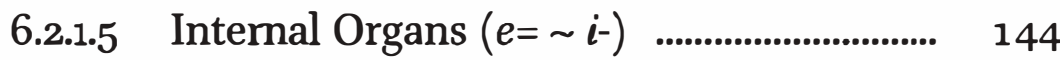

6.2.1.6 Nodular or Curved Structure $(a r a=, r a=) \quad \ldots \quad 145$

6.2.1.7 Lower Parts of Body $(o=\sim \mathrm{j}=)$.................. 146

6.2.2 Is There a Hierarchy? ..................................................... 148

6.3 Kinship Terms .................................................................................... 149

6.3.1 Parallels between the Body Part Terminology and Kinship Terms ................................................................ 150

6.4 The Twin Levels ...................................................................................... 150

6.5 Secondary Possession .............................................................. 151

6.5.1 Juxtaposition/Compounds ............................................. 151

6.5.2 Complex Structures ......................................................... 152

6.5.2.1 Lexical Compounding with

Class Markers ................................................... $\quad 152$

6.5.2.2 Double Marking and Clitic Sequencing 153

6.5.2.3 Adverbial Function ......................................... 153

6.5.2.4 Double Class Markers ................................ 155

6.5.2.5 Summary ........................................................ 156

6.6 Animate vs. Inanimate Possessor and the Semantics of

Inalienability ................................................................................... $\quad 156$

6.6.1. Inanimate ........................................................................... 156

6.6.2 Alienated but Inherent .............................................. 157

6.6.3 Part-to-Whole or Part-to-Component ....................... 159

6.6.4 Inalienable Possessed Nouns: A Conspectus ........ $\quad 160$

$6.7 \quad$ Alienable Nouns ...................................................................................... 163

6.7.1 Possessed Nouns ................................................................... 163

6.7.2 Twelve Different Varieties ........................................... 163

6.8. Attributive Modification and Possession ............................. $\quad 165$

6.9. The "possessive" Relationship: The 'Have' Construction ..... 166

6.10 Conclusion ...................................................................................... 167 
VII. Pronouns, Pronominal and Object Clitics

7.1 Personal Pronouns ........................................................................... 169

7.1.1 Person and Number $\quad$........................................................ $\quad 169$

7.1.2 Second Person Pronouns and Honorifics .............. 171

7.2 Demonstrative Pronouns ......................................................... 172

7.3 Pronominal Clitics .................................................................... 173

7.3.1 Human Arguments (Subject and Object) .............. 175

7.3.2 Object Clitics .................................................................... 176

7.3.2.1 Pronominal Objects ....................................... 176

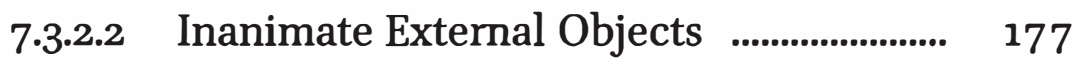

7.3.2.3 Internalised Objects .................................... $\quad 178$

7.3.3 Pronominal Clitics and Temporal Adverbs $\quad$.......... $\quad 179$

7.4 Interrogative and Indefinite Pronouns ................................ 180

7.5 Reflexive Forms ........................................................................ 182

7.6 Reciprocal Pronouns .................................................................. 186

7.7 Locational Adverbial Demonstratives $\quad$.................................. $\quad 187$

VIII. Modification Including Temporal and Spatial Deixis Introduction .............................................................................................. $\quad 189$

8.1 Adjectives .......................................................................................... 189

8.1.1 Characteristic Features _................................................ 189

8.1.2 The Semantic Content ................................................. 190

8.1.3 Attributive Adjectives ................................................. 190

8.1.4 Predicative Adjectives ................................................... 191

8.1.5 Colour Terms .................................................................... 193

8.2 Dependency of Modifiers ....................................................... 193

8.2.1 Proclitics and Modification ........................................... 193

8.2.2 Non-Dependent Modifiers ......................................... 197

8.3 Manner Adverbs .......................................................................... 197

8.4 Temporal Adverbs and Temporal Deixis ............................ 199

8.4.1 Subjecthood and Temporal Adverbs ....................... 199

8.4.2 Syntax of Words Indicating Time ............................ $\quad 200$

8.4.3 Compounding and Lexicalisation $\quad$............................. 202

8.4.4 Multiplicity of Temporal Deixis ................................ 202

8.4.5 Celestial Bodies as Temporal Markers .................... 203

8.4.6 Temporal Categorisation and Hunting and Gathering ......................................................................... $\quad 203$

8.5 Spatial Deixis .................................................................................... $\quad 204$

8.5.1 Distance and Direction ................................................ $\quad 205$

8.5.2 Vertical and Horizontal Space ................................... $\quad 206$

8.5.3 Locational/Spatial Postpositions $\quad$................................ $\quad 207$ 
8.6 Body Division Classes and Adverbs ………............................ 209

8.7 Third Person Demonstrative Pronouns and Deixis ........... 209

IX. The Verb and Verb Complex

Introduction

9.1 Verbal Proclitics _........................................................................ 214

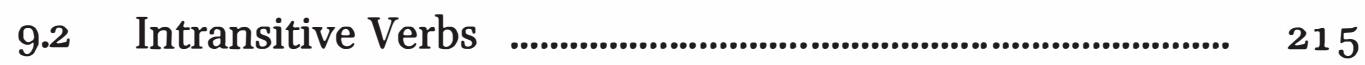

9.3 Transitive Verbs ....................................................................... 217

9.4 Reflexive/Self-Directed ................................................................ 223

9.5 The Distancing of the Verbal Proclitic from its Host ...... 224

9.6 The Causative/Applicative ........................................................ $\quad 226$

9.6.1 The Morphological Causative .................................... 227

9.6.2 Periphrastic Causatives ............................................. 230

9.7 Formative Affixes ......................................................................... 231

9.8 Tense, Aspect and Mood (TAM) ................................................. 233

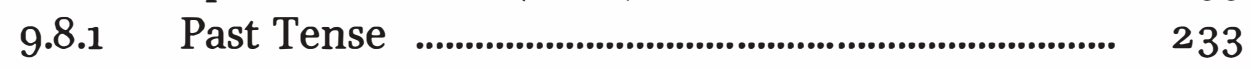

9.8.2 Non-Past Tense _........................................................... 236

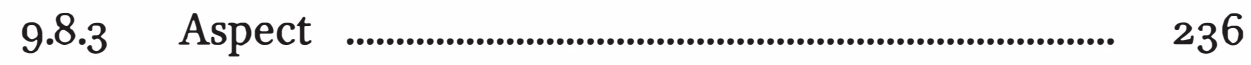

9.8 Mood ............................................................................. 238

9.9 The Copula Be or Jiyo .............................................................. 240

9.10 The Verbs 'Come' and 'Go' .............................................................. 241

X. Syntactic Organisation

Introduction

10.1 Word Order

10.1.1 Adjectives and Nouns _................................................. 243

10.1.2 Genitive ...................................................................... 245

10.1.3 Case Markers .................................................................... 245

10.1.4 Numerals and Quantifiers $\quad$.......................................... 246

10.1.5 Degree Words ................................................................... 246

10.1.6 Manner Adverbs ......................................................... 247

10.1.7 Temporal Adverbs .................................................... 247

10.1.8 Negative Verb ................................................................... 248

10.1.9 Relative Markers ......................................................... 248

10.1.10 Interrogatives ............................................................................ 249

10.1.11 Adpositional Phrases .................................................... 249

10.1.12 Variability in Word Order ............................................ 250

10.1.13 Deviation from the Standard SOV Pattern .......... 250

10.1.14 Variability in Proclitics ............................................... 250 
10.2 Coordination .............................................................................. 251

10.2.1 Nominal Coordination ...................................................... $\quad 252$

10.2.2 Pronominal Coordination ............................................ $\quad 252$

10.2.3 Adjectival Coordination ............................................. $\quad 252$

10.2.4 Verbal and Sentential Coordination .......................... 253

10.2.5 Adversative Coordination ............................................ 253

10.2.6 Comitative Conjunct ................................................... 254

10.3 Negation ............................................................................................................ 254

10.3.1 Emphatic Negative Coordination ............................ 255

10.3.2 Prohibitive Negation ............................................................ 255

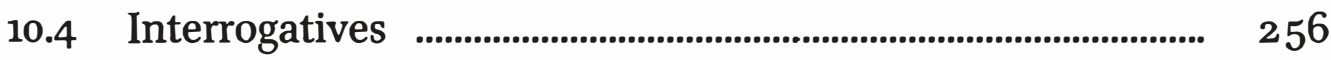

10.5 Non-Finite Subordination $\quad$..................................................................... $\quad 258$

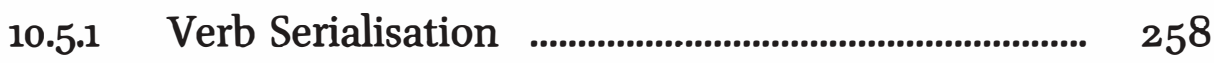

10.5.2 Cause and Effect .................................................................. 261

10.5.3 Conditional Clauses ........................................................ 261

10.5.4 Action Nominals .......................................................... $\quad 262$

10.6 Clause Chaining ..................................................................................... $\quad 263$

10.7 Comparative Constructions ....................................................... 264

10.8 Relativisation .................................................................................. 267

10.9 Syntax of the Possessive Construction ................................... $\quad 269$

10.9.1 Possessed NP as the Head .......................................... $\quad 269$

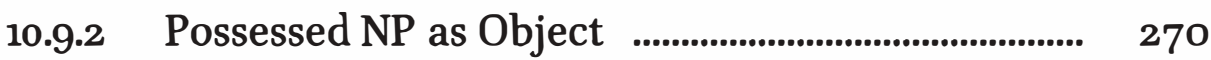

10.9.3 Identification Question ..................................................... $\quad 270$

10.9.4 Complement Phrase ..................................................... 270

10.9.5 Verbless Constructions ................................................ 270

Appendix A: Lico's Genealogical Affiliation and Her Language

Profile

Appendix B: An Acoustic Study of Problematic Laterals .................. $\quad 275$

Text: The Great Narrative of Phertajido .................................................... $\quad 279$

Bibliography ................................................................................................... 291

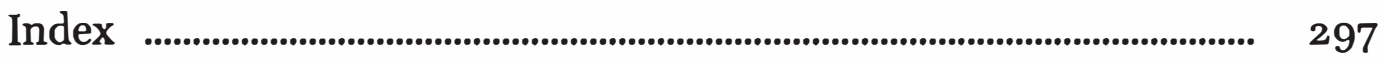

Photographs of Contact Persons 


\section{ACKNOWLEDGEMENTS}

First of all, I have to express deep gratitude to the Great Andamanese people, who made it possible for me to explore the moribund language during my fieldwork from 2005 to 2009. The native speakers who helped me collect first-hand data, especially those who spent long hours with me on Strait Island and in the city of Port Blair are: Nao Jr., Boa Sr., Lico, Peje, and Noe. I am very thankful to them and others for their patience and willingness to show me the jungles, marshes, seashores, their dwellings and burial places in the Strait, and places in Mayabunder. This enabled me to collect a large number of relevant lexical items on location. Most importantly, the members of the tribe accepted me as a family member and this bond exists till date. But for the cooperation and understanding of the Great Andamanese tribe, I could not have even begun to think of writing this grammar. Their contribution to this work is greatly appreciated.

I thank my sounding boards, Bernard Comrie, John Peterson, Balthasar Bickel, Andrew Spencer, Tania Kuteva, Ayesha Kidwai and Pramod Pandey. Their suggestions and criticism have helped me in the analysis of a very unique language. I also wish to take this opportunity to thank Samantha Goodchild, Karen Buseman and Alan Buseman, in helping me prepare the final draft. Any errors that remain are mine alone.

I am very grateful to two anonymous referees for providing me detailed comments and suggestions on a draft version of this book. Their laudable comments on the first version inspired me to prepare the final copy for publication.

I am most grateful to Andrej Malchukov for reading parts of this grammar and for giving insightful comments and suggestions. The students associated with the project VOGA deserve special thanks for assisting me whenever required. Special mention should be made of: Pramod Kumar, Alok Das, Bidisha Som, Abhishek Avatans, Mayank, Narayan Chaudhary and Sandy Ritchie.

I also acknowledge the help at various levels provided by the Ministry of Human Resources and Development (MHRD), the Ministry of Tribal Welfare, the Andaman Adim Jan Jati Vikas Samiti (AAJVS), the Jawaharlal Nehru University, New Delhi and the School of Oriental and African Studies, University of London, UK. 
Books like this one cannot be completed without the assistance and generosity of the funding agency, viz. ELAR, SOAS, University of London, which granted me funds for the mega project Vanishing Voices of the Great Andamanese (VOGA) www.andamanese.net and http://elar.soas.ac.uk/ deposit/abbi2oo6greatandamanese.

This grammar was written at three different places while I was on long leave from my university. I would like to express my gratitude to Bemard Comrie who provided me the right atmosphere at the Max Planck Institute of Evolutionary Anthropology, Germany where I commenced writing this grammar. I am thankful to Alexandra Aikhenvald who invited me as a Visiting Professor at James Cook University at Caims, Australia so that I could work on this grammar. I am grateful to her and Robert Dixon for the discussion on the main tenets of the grammar of Great Andamanese. I am also thankful to Barbara Lotz for providing me a peaceful environment at the University of Würzburg, Germany, to give the final touches to the manuscript.

I am greatly indebted to Peter Austin of SOAS, University of London, UK where I was associated as the Leverhulme Professor for nine months for the provision of both physical and mental space to think and complete the grammar. But for the help that he and his staff offered I could not have accomplished the task at hand. I dedicate this work to him.

I will be indebted all my life to Satish Abbi, my husband, who not only encouraged me to plunge into this venture but also accompanied me to the field as much as possible. His help, understanding, and cooperation kept the fire buming in me despite many official, physical and psychological hurdles.

I feel very happy and satisfied that I could accomplish the task of writing this grammar before the joumey of the language into oblivion.

Jawaharlal Nehru University

New Delhi 


\section{PREFACE}

This grammar is the result of a major language documentation project Vanishing Voices of the Great Andamanese (VOGA), which was undertaken from 2005 to 2009 in the Andaman Islands. Under the auspices of the Hans Rausing Endangered Languages Project, I, along with a team of research assistants spent approximately 40 months in the Andamans, documenting Great Andamanese and producing descriptive and theoretical work on the language. The team members spent varying amounts of time on the island during this four-year period, but I spent the longest as after the initial phase of fieldwork, my research assistants could not sustain their interest in the work primarily because of the threatening attitude of some of the officials, the difficult living conditions in the islands and boredom.

Present-day Great Andamanese (PGA) is a highly endangered language; when the VOGA team first visited Strait Island, the current home of the tribe, there were nine speakers. By the time the team left the Andamans for the last time in 2009 there were only six left. The last speaker of Bo, a variety of Great Andamanese, also died in January 2010. At present there are only five speakers with varying degrees of competence in the language. PGA is a complex and diverse language; it is a koiné formed from four surviving northern Great Andamanese languages: Khora, Jeru, Sare and Bo. It draws its lexicon from all four of these dialects but its grammar is primarily based on Jeru. It displays highly unusual grammatical features such as body part proclitics modifying all parts of speech. Perhaps most importantly, PGA is a unique language; there is strong linguistic and genetic evidence to suggest that the people of the Andaman Islands represent a distinct genetic group who populated the islands from the mainland tens of thousands of years ago. According to some geneticists, Andamanese are the survivors of the first migration out of Africa 70.000 years BP. The language is a fast-closing window on a very ancient form of cognition, or as Nicholas Ostler puts it in his review of the A Dictionary of the Great Andamanese Language. English-Great Andamanese-Hindi (Anvita Abbi 2012): "700 centuries of unique experience terminated in just two."

In addition to the Great Andamanese, there are three other distinct indigenous ethnolinguistic groups inhabiting the Andamans: the Jarawa, the Onge and the Sentinelese. My first introduction to the tribes of the Andaman Islands was made in 2000-2001 when I conducted a pilot survey 
of the languages of the island titled Language Survey of Andaman supported by the Max Planck Institute of Evolutionary Anthropology, Leipzig, Germany. The results of this survey not only opened the road to language documentation but also paved the path to explore the grammar of the Great Andamanese language.

All the languages spoken by these peoples are endangered as their population bases dwindle and Hindi extends its reach ever further in the islands. Great Andamanese is the closest to extinction, however; today only two of the four northem varieties of the language survive. The other two, Khora and Bo, became extinct with the passing of their last speakers Boro Sr. and Boa Sr. respectively.

Little is known about the affairs of the inhabitants of the Andaman Islands until the arrival of the British in the 186os. The early colonists reported around 5000 people living as hunter-gatherers. At that time there were ten distinct varieties of Great Andamanese named after the ten tribes which spoke them. An estimated 3000-3500 Great Andamanese people lived right across the group of islands known as the Great Andaman. By the time of the 1901 Census of the islands, this number had fallen to just 625. It seems that contact with mainlanders had a devastating effect on the indigenous population, as Edward Horace Man noted: "The interesting Negrito race inhabiting the Andaman Islands is doomed to early extinction-save possibly the small section occupying Little Andaman." (27th June 1918).

By the time of the VOGA team's arrival in 2005, this ominous prediction had sadly been borme out; the number of the Great Andamanese had dwindled to just 51 , with only ten members of the tribe still able to speak some form of the language. When the team first encountered the people, they found their lives further blighted by the 2004 tsunami, which drove them from their homes in Strait Island and forced them into temporary shelters in the Andaman capital of Port Blair. Many of the earlier recordings were made in this difficult period. The people were often withdrawn and unwilling to collaborate with the VOGA team, preoccupied as they were with dealing with the difficult situation in which they found themselves. In late 2005 the members of the tribe returned to their homes in Strait Island, a small island to the northeast of Port Blair. The later recordings were all made there.

The Great Andamanese of today live on subsidies provided to them by the Indian government, though some members still continue their traditions of fishing for crabs and other sea-life and hunting turtles. Gathering tubers and potatoes, however, is now virtually non-existent. Most tribe 
members understand Hindi, Bangla and a few words of English as well as some words in Great Andamanese. Children under 16 now do not speak or understand the heritage language. The main language of communication amongst members of the tribe is the Andaman variety of Hindi.

The unusual structures of the language captured in the present grammar motivate us to capture the 'possible' structures of human language.

SOAS, University of London, UK 



\section{LIST OF MAPS, FIGURES AND TABLES}

\section{MAPS}

\begin{tabular}{|c|c|}
\hline & Southeast Asia \\
\hline & Location of the Andaman Islands \\
\hline & The 19th-2oth century \\
\hline & The Strait Island \\
\hline & Distribution of the Andaman tribes \\
\hline
\end{tabular}

\section{FIGURES}

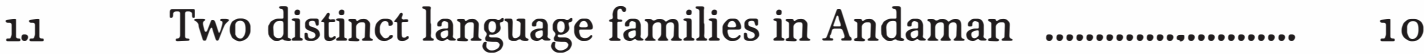

1.2 Present Great Andamanese and its regional varieties ........ 11

1.3 The number and age of the Great Andamanese people (2005-2006) ........................................................................................... 20

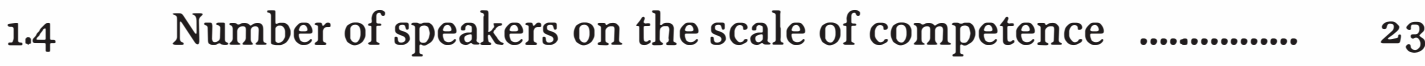

1.5 Age-wise competence level of the speakers in $2007 \quad \ldots . . . . . . . . . .23$

1.6 The number and mean age of the speakers on the competence scale ........................................................................................... 24

2.1 Syllable breaks _................................................................................ $\quad 57$

3.1 Degree of grammaticalisation across grammatical categories

3.2 The classificatory functions of body class markers in PGA

6.1 Body division classes and possession .......................................... 162

6.2 Word formation processes involved in possessive constructions

$\mathrm{Al} \quad$ Lico's genealogical affiliation and her language profile $\quad . . . . \quad 273$

B1a-b The spectrogram of $l \varepsilon: c$ 'arrow' as articulated by Peje ........ $\quad 276$

B2a-b The spectrogram of $l \varepsilon: c$ 'arrow' as articulated by

Nao Jr.

276

B3 The formant structure of $[1]$ (Peje) ............................................... 277

B4 The formant structure of [l] (Nao Jr.) .................................... 277 


\section{TABLES}

1.1 The Andamanese languages in the 19th century ........................ 10

1.2 Comparative lexicon in Angan and Great Andamanese ......... 15

1.3 The lineage of the oldest and the best speakers ......................... 21

$1.4 \quad$ Lineage of the semi-speakers ................................................................... 21

1.5 Stages of naming an individual ......................................................... 29

1.6 Honey calendar or names of the seasons ....................................... 30

1.7 Blooming of flowers and associated months of the year ........ 31

1.8 Measuring time in PGA ......................................................................... 33

2.1 Vowels of Great Andamanese ........................................................... 37

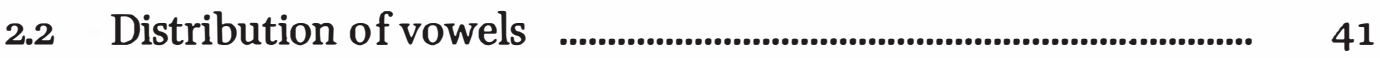

2.3 Vowel clusters in PGA .......................................................................... 42

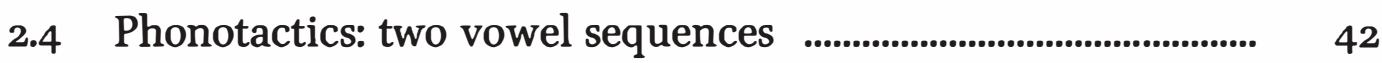

2.5 Intervocalic semi-vowels .................................................................... 44

2.6 Words that end in semi-vowels ............................................................. 45

2.7 Words that begin with semi-vowels ..................................................... 46

2.8 Intra-community variation of unusual sounds ............................. 46

2.9 Phonetic variation across community members $\quad$......................... 47

2.10 Consonants of Great Andamanese .................................................. 48

2.11 Distribution of consonant sounds $\quad$..................................................... 53

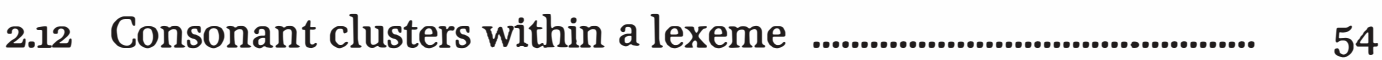

2.13 Geminates ......................................................................................................... 56

2.14 Possible syllable structures $\quad$.................................................................. 58

3.1 Seven basic zones in the partonomy of the body ....................... 80

3.2 Semantics of body division class markers and bound form classes

4.1 Spatial words and the combination of clitics and affixes $\quad . . . . . . \quad 103$

4.2 Left-headed Compounds .......................................................................... 105

4.3 Right-headed Compounds ...................................................................... 106

4.4 Non-compositional compounds ........................................................ 107

4.5 Compositional compounds ...................................................................... 107

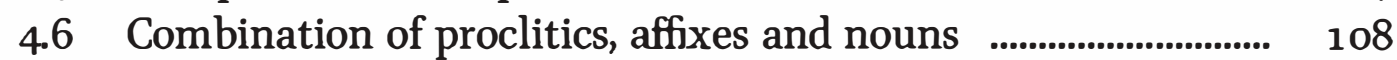

5.1 Case markings ......................................................................................... $\quad 117$

5.2 Locative forms ..................................................................................................... $\quad 127$

6.1 Seven basic zones in the partonomy of the body ...................... 140

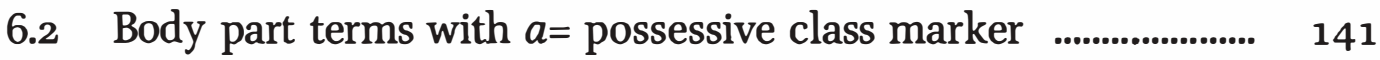

6.3 Body part terms with $e=-, \varepsilon r=$ possessive class marker $\ldots . . . . . . . . . . \quad 141$

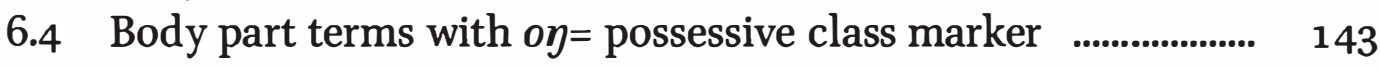

6.5 Body part terms with $o t=$, ot $=$, $u t=$ possessive class marker $\quad \ldots \quad 144$ 
6.6 Body part terms with $e=, i=$ possessive class marker

6.7 Body part terms with $a r a=$ possessive class marker .................. $\quad 146$

6.8 Body part terms with $0=, 0=$ possessive class marker ................ $\quad 147$

6.9 Frequency of occurrence of body division class markers with body part terminology $\quad$............................................................................... $\quad 148$

6.10 Kinship terms .................................................................................. 149

6.11 Parallels between body parts and kinship terms ........................ 150

6.12 Double marking and secondary possession .................................... $\quad 154$

6.13 Terms for the parts of a tree or a plant ........................................... 160

6.14 Possessive class markers defining primary possession and classes of nouns ....................................................................................... 161

6.15 Varieties of possession .......................................................................... $\quad 165$

7.1 Pronominal forms in PGA ..................................................................... 169

7.2 Pronominal clitics ..................................................................................... 174

7.3 Indefinite/interrogative forms _.......................................................... 181

8.1 Body division classes in adjectives .................................................. 194

8.2 Edge and side of an object ...................................................................... 197

8.3 Multiplicity of temporal deixis ....................................................... 202

8.4 Terms for direction and days of a month ....................................... $\quad 203$

8.5 Names of flowers and hunting time ..................................................... 204

8.6 Levels of the sea ....................................................................................... 205

8.7 Landscape and coastal terms ……………............................................. $\quad 205$

8.8 Spatial deixis describing parts of a Great Andamanese house .............................................................................................................. 206

8.9 Spatial adverbs and their morphemic composition ................... 208

8.10 Body division classes designating spatial relations .................. 209

8.11 Body division classes in adverbs ....................................................... 210

8.12 Demonstrative pronouns ....................................................................... 211

9.1 The body division class markers with verbs ................................. $\quad 215$

9.2 Verbs with Formative affixes, tense and mood suffixes .......... $\quad 239$

9.3 Verbal affixes in PGA ............................................................................... $\quad 241$ 



\section{ABBREVIATIONS AND SYMBOLS}

\begin{tabular}{ll}
1 & first person \\
2 & second person \\
3 & third person \\
A & agent \\
ABL & ablative \\
ABS & absolutive \\
ACC & accusative \\
ADJ & adjective \\
ADV & adverb \\
AGT & agentive \\
APPL & applicative \\
C & consonant \\
CAUS & causative \\
CL 1, CL 2, etc. & body division class 1, 2, etc. \\
CLASS & classifier \\
CLT & clitic \\
COM & comitative \\
COMP & complementiser \\
COMPR & comparative \\
CONJ & conjunct \\
COP & copula \\
COND & conditional \\
CONV & converb \\
D & possessed \\
DAT & dative \\
DEM & demonstrative \\
DIR & directional \\
DIS.VIS & distant visible \\
DIS.INVIS & distant invisible \\
DST.PST & distant past \\
DO & Direct object \\
DU & dual \\
EMPH & emphatic \\
ERG & ergative \\
EXCL & exclusive \\
& \\
\hline
\end{tabular}


xxvi

\begin{tabular}{|c|c|}
\hline EXIST & existential \\
\hline $\mathrm{F}$ & female \\
\hline FA & formative affix \\
\hline GEN & genitive \\
\hline $\mathrm{H}, \mathrm{HON}$ & honorific \\
\hline HAB & habitual \\
\hline IMM.PST & immediate past \\
\hline IMP & imperative \\
\hline IO & indirect object \\
\hline INA & inalienability \\
\hline INCL & inclusive \\
\hline INSTR & instrument \\
\hline INT & intimate \\
\hline LOC & locational \\
\hline M & male \\
\hline MOD & modifier \\
\hline MT & mother tongue \\
\hline $\mathrm{N}$ & noun \\
\hline NEG & negative \\
\hline NMLZ & nominaliser \\
\hline NOM & nominal \\
\hline NP & noun phrase \\
\hline NPST & non-past tense \\
\hline NRR.PST & narrative past \\
\hline $\mathrm{O}$ & object \\
\hline OBJ & object clitic \\
\hline PGA & Present-day Great Andamanese \\
\hline PCPL & participial \\
\hline PL & plural \\
\hline PP & postpositional phrase \\
\hline POSS & possessive \\
\hline PRO & pronoun \\
\hline PROHB & prohibitive \\
\hline PROX & proximate \\
\hline PROX1 & proximate, nearer to the speaker \\
\hline PROX2 & proximate, nearer to the hearer \\
\hline PST & past \\
\hline Q & question \\
\hline R & possessor \\
\hline
\end{tabular}




$\begin{array}{ll}\text { REC } & \text { reciprocal } \\ \text { REL } & \text { relativiser } \\ \text { REFL } & \text { reflexive } \\ \text { RESULT } & \text { resultative } \\ \text { S } & \text { subject } \\ \text { SG } & \text { singular } \\ \text { Sp } & \text { speaker } \\ \text { SPEC } & \text { specific } \\ \text { STAT } & \text { stative } \\ \text { SUBJ } & \text { subjunctive } \\ \text { TAM } & \text { tense, mood and aspect } \\ \text { TR } & \text { transitiviser } \\ \text { V } & \text { verb } \\ \text { Vt } & \text { verb transitive } \\ \text { Vi } & \text { verb intransitive } \\ \text { X } & \text { any entity } \\ \text { F } & \text { clitic boundary }\end{array}$

